\title{
3-Dimensional Nanoscale Structural Characterization of Magnetic Tunnel Junction
}

\author{
Y. Z. Liu ${ }^{*}$, A. K Petford-Long ${ }^{*}$, D. K Schreiber ${ }^{* * *}$, Y-S Choi ${ }^{* * *}$, D. Djayaprawira ${ }^{* * *}$, D N Seidman ${ }^{* *}$
}

${ }^{*}$ Materials Science Division, Argonne National Laboratory, Argonne, Illinois 60439

** Department of Materials Science and Engineering, Northwestern University, Evanston, Illinois 60208

${ }^{* * *}$ Electronic Devices Engineering Headquarters, Canon ANELVA Corp., Kanagawa, Japan

A magnetic tunnel junction (MTJ) consists in its simplest form of two ferromagnetic layers separated by a thin insulating layer such as $\mathrm{MgO}$. Despite being used commercially as hard disk drive read heads and solid state memory devices, MgO-based MTJs [1] are not well understood. Indeed, current state-of-the-art MTJs achieve only a fraction of their theoretical performance capabilities. To better understand the microstructural origins of the magnetotransport properties, the three-dimensional (3D) morphology and chemistry of the individual layers and their interfaces of a simple MTJ structure $(\mathrm{CoFe}(10) / \mathrm{Ru}(0.8) / \mathrm{CoFeB}(3) / \mathrm{MgO}(1.2) / \mathrm{CoFeB}(3) / \mathrm{Mg}(1) / \mathrm{CoFe} \mathrm{Cap}$, layer thicknesses in $\mathrm{nm}$ ) were mapped with high spatial resolution using STEM and EFTEM electron tomography and atom-probe tomography (APT), both before and after annealing at $360^{\circ} \mathrm{C}$ for 2 hours.

A dual-beam focused ion-beam (FIB) system was used to prepare cylindrical specimens with a diameter of $\sim 60 \mathrm{~nm}$. Fig. 1 shows a TEM tomography sample of the annealed MTJ. Figure 2 shows the good crystallinity in the $\mathrm{MgO}$ barrier and $\mathrm{CoFeB}$ layers, and also the morphology of the specimen (insert in Fig. 2). A 1D elemental profile across the same MTJ from APT is shown in Fig. 3. During annealing, B diffused away from the $\mathrm{MgO}$ barrier as the $\mathrm{CoFeB}$ crystallized.

The 3D morphology of the MTJs was analyzed using z-contrast electron tomography in STEM mode and EFTEM tomography. Figure 4 shows a SIRT method reconstruction [2] from a single tilt series, collected from $-80^{\circ}$ to $+80^{\circ}$ with step of $1^{\circ}$ using the high angle annular dark field (HAADF) detector. The layered structure and grain distribution in CoFe cap layer are clearly seen. The interfaces on either side of the tunnel barrier are clearly rough, as is the interface above the upper ferromagnetic layer, and this will play a significant role in the resulting magnetotransport properties of the MTJ and thus in the ultimate device performance.

The electron microscopy was carried out at the Electron Microscopy Center, Argonne National Laboratory, a U.S. Department of Energy Office of Science Laboratory operated under Contract No. DE-AC02-06CH11357 by UChicago Argonne, LLC. Atom-probe tomography measurements were performed at the Northwestern University Center for Atom-Probe Tomography (NUCAPT).

References

[1] S. Yuasa and D.D. Djayaprawira, J. Phys. D: Appl. Phys. 40, R337-R354 (2007).

[2] P. Gilbert, J. Theor. Biol. 36 (1972). 105. 


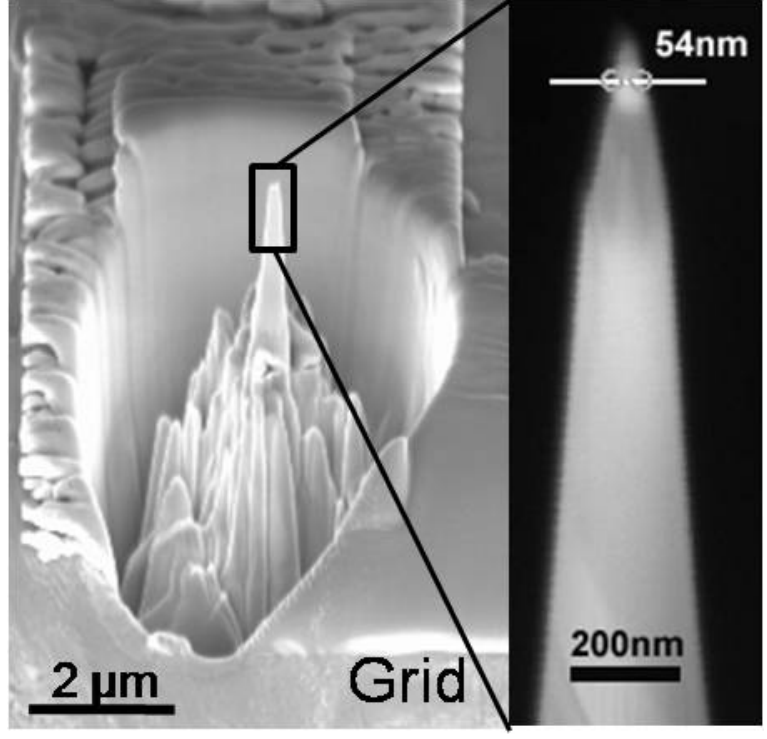

Fig. 1. FIB images of cylindrical tomography specimen. The left image shows the fabricated sample on the grid and the right shows the specimen tip. (Sample diameter $\sim 54 \mathrm{~nm}$ )

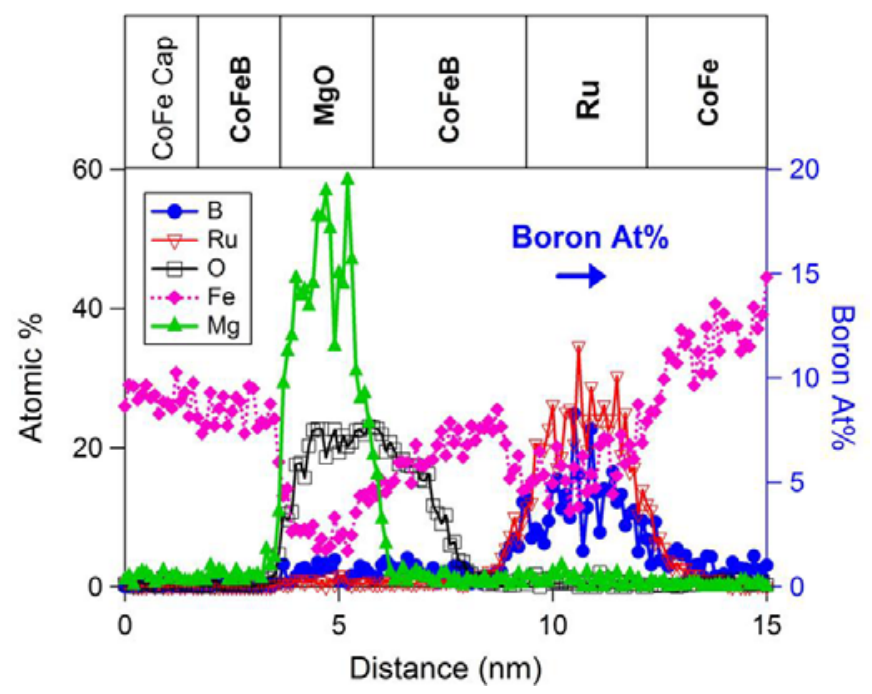

Fig. 3. 1D composition profile from APT data, showing that $\mathrm{B}$ has diffused away from the $\mathrm{MgO}$ after annealing. Note that the high $\mathrm{Mg}$ content in the barrier is the result of diffusion from the $\mathrm{Mg}$ cap layer to the $\mathrm{MgO}$.

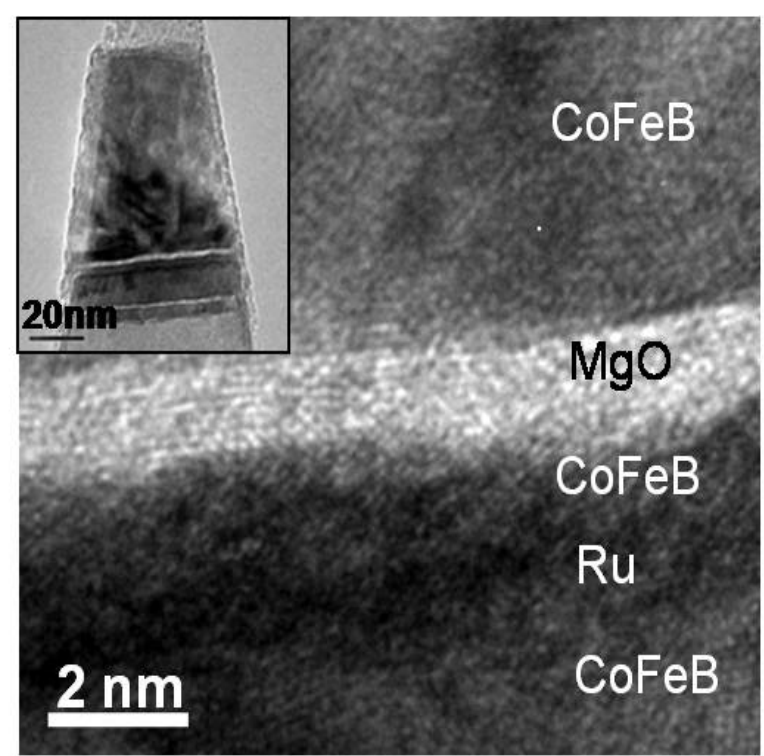

Fig. 2. HREM image shows good crystallinity in the $\mathrm{MgO}$ barrier and $\mathrm{CoFeB}$ layers after annealing. Top-left insert shows the sample morpholology.

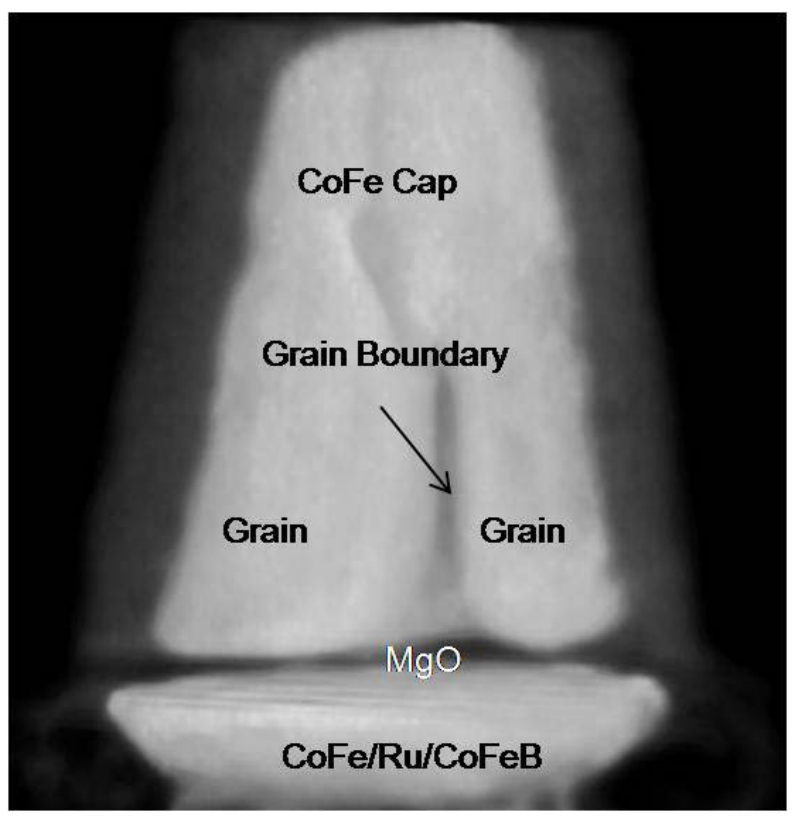

Fig. 4. Projection of reconstructed STEM tomography data. The MTJ layers and the grains in the CoFe cap layer are shown clearly in 3D. 